\title{
Nutrient-wide association study of 92 foods and nutrients and breast cancer risk
}

\author{
Alicia Heath ${ }^{1}$, David Muller ${ }^{1}$, Piet van den Brandt ${ }^{2}$, Nikos Papadimitriou ${ }^{3,4}$, Elena Critselis ${ }^{5}$, \\ Elisabete Weiderpass ${ }^{4}$, Elio Riboli ${ }^{1}$, Konstantinos Tsilidis ${ }^{1}$, Ioanna Tzoulaki ${ }^{1}$ and on behalf of \\ the EPIC investigators ${ }^{4}$ \\ ${ }^{1}$ Imperial College London, London, United Kingdom, \\ ${ }^{2}$ Maastricht University Medical Centre, Maastricht, Netherlands, \\ ${ }^{3}$ University of Ioannina School of Medicine, Ioannina, Greece, \\ ${ }^{4}$ International Agency for Research on Cancer, Lyon, France and \\ ${ }^{5}$ Biomedical Research Foundation of the Academy of Athens, Athens, Greece
}

\begin{abstract}
Several dietary factors have been extensively investigated for associations with risk of breast cancer, but to date unequivocal evidence only exists for alcohol consumption. We sought to systematically evaluate the association between 92 dietary factors and breast cancer risk using a nutrient-wide association study approach. Using data from 272,098 women participating in the European Prospective Investigation into Cancer and Nutrition (EPIC) study, we assessed dietary intake of 92 foods and nutrients estimated by dietary questionnaires. Cox regression with age as the time scale and adjustment for potential confounders, was used to quantify the association between each food or nutrient and risk of breast cancer. A false discovery rate (FDR) of 0.05 was used to select the set of foods and nutrients to evaluate in the independent replication cohort, the Netherlands Cohort Study (NLCS). During a median follow-up time of 15 years, 10,979 incident invasive breast cancers were identified in the women from the EPIC study. Six foods and nutrients were associated with risk of breast cancer when controlling the FDR at 0.05 . Higher intake of alcohol overall was associated with a higher risk of breast cancer (hazard ratio (HR) for a $1 \mathrm{SD}$ increment in intake $=1.05,95 \%$ confidence interval (CI) $1.03-1.07$ ), as was beer/ cider intake and wine intake (HRs per 1 SD increment $=1.05,95 \%$ CI $1.03-1.06$ and $1.04,95 \%$ CI $1.02-1.06$, respectively), whereas higher intakes of fibre, apple/pear, and carbohydrates were associated with a lower risk of breast cancer (HRs per $1 \mathrm{SD}$ increment $=$ $0.96,95 \%$ CI $0.94-0.98$; 0.96, 95\% CI $0.94-0.99$; and $0.96,95 \%$ CI $0.95-0.98$, respectively). When evaluated in the NLCS (2368 invasive breast cancer cases), estimates for each of these foods and nutrients were similar in magnitude and direction, with the exception of beer/cider intake, which was not associated with risk of breast cancer in the NLCS. Our findings confirm the well-established increased risk of breast cancer associated with alcohol consumption, and suggest that higher intake of dietary fibre, and possibly fruit and carbohydrates, might be associated with reduced breast cancer risk.
\end{abstract}

\section{Conflict of Interest}

There is no conflict of interest 